Im vorstehenden hoffe ich ziemlich zuverlässige Formeln für die Korrektion der in meiner frutheren Arbeit gegebenen Koordinaten der Sterne im Sternhaufen Messier I $_{3}$ abgeleitet zu haben. Freilich muß man sich darttber klar sein, $\mathrm{da} ß$ bei Vergleichung zukünftiger Messungen eines Sternhaufens mit solchen, die jetzt angestellt worden sind, für die letzteren doch stets neue Reduktionselemente berechnet werden dürften. Denn die Positionen und Eigenbewegungen der Vergleichsterne werden meist in Zukunft genauer bekannt sein, als sie es jetzt sind. Die Hauptaufgabe bei der Ausmessung eines Sternhaufens ist daher meines Erachtens zur Zeit die, daß man die relativen Koordinaten in einem fest definierten Koordinatensystem möglichst genau ermittelt *) und die Ergebnisse in einer Form mitteilt, daß eine spätere Verbesserung auf Grund neuer Reduktionselemente leicht mög. lich ist. Wenn letztere schon jetzt möglichst scharf bestimmt sind, so wird dies dem späteren Bearbeiter seine Aufgabe erleichtern, ist aber im tubrigen von sekundärer Bedeutung.

Potsdam, Astrophysikalisches Observatorium, 1908 Juli 4.

H. Ludendorff.

*) Ich möchte bei dieser Gelegenheit darauf hinweisen, daß bei der Ausmessung enger Sternhaufen die von Kostinsky (Mitteil. der Nikolai-Hauptsternwarte zu Pulkowo, Bd. II, Nr. 14) konstatierte Einwirkung zweier eng benachbarter photographischer Sternscheibchen aufeinander die Genauigkeit beeinträchtigen kann.

\title{
Das Spektrum des Milchstraßennebels H V 15 Cygni.
}

Der langgestreckte, ziemlich schmale Milchstraßennebel H V ${ }_{15}$ Cygni $=$ NGC. 6960 verdient deshalb besonderes Interesse, weil er in ganz ausgesprochener Weise an der Höhlenbildung der Milchstraße beteiligt ist. Fast genau von Süd nach Nord ziehend, scheidet er scharf eine Gegend größter Sternfülle im Osten von einer solchen geringer Sternzahl im Westen. Ich habe ihn deshalb fruher als einen »Randnebels bezeichnet. Zur selben Gattung gehören die Nebel bei $\zeta$ Orionis und $\psi$ Eridani. Roberts beschreibt den Nebel in seinem ersten Bande p. 115: It is more than two degrees in length from $\mathbf{N}$ to $S$ and forty-seven minutes of arc in breadth on the following side of $52(k)$ Cygni, which it barely touches by a slight projection of the nebulosity. The streaky character of the nebulosity will be observed on the print, and when an opportunity occurs to take another photograph with a much longer exposure of the plate, this connection of several parts of the nebulosity will doubtless be shown. ...

In der Tat hängt der Nebel, wie die Aufnahmen mit meinem Sechszöller im Juni $r 891$ ergeben haben, sogar mit dem Nebel H V I4 Cygni = NGC. 6992 innig zusammen.

Aufnahmen mit dem Reflektor zeigten im vergangenen Jahre, da $B$ an verschiedenen Stellen fast zweifelfreie Verbindungen zwischen den Nebelstreifen und einzelnen Sternen nachweisbar sind, so daß der Nebel als in der Tiefe zahlreicher Milchstraßenketten liegend angesehen werden darf.
Es war daher von besonderem Interesse, herauszubringen, ob dieser Nebel kontinuierliches Spektrum oder Gasspektrum besitzt.

Am 3. August 1908 gelang mir mit dem Waltz-Reflektor eine $3^{1} / 2$-stündige Spektralaufnahme derjenigen Teile des Nebels, die nördlich von 52 Cygni zu großer Intensität auf schmalen Raum zusammengedrängt sind.

Es ergab sich ein ausgesprochenes Gasspektrum.

Weitaus die hellste Linie liegt am violetten Ende des Spektrums. Die anderen Linien sind viel schwächer, so daß ich unter dem Mikroskop nicht auf sie einzustellen vermochte. Ich halte nach verschiedenen Messungsversuchen die helle Linie für die Nebellinie $\lambda=373$

Unter dieser Annahme wären wohl die schwachen Linien zu identifizieren; nämlich $\lambda=434$, dann das zusammengewaschene Band der beiden ersten Hauptlinien um $\lambda=500$, und die Linien $\lambda=369,397$ und 411 und zwar bezuglich ihrer Intensität vielleicht in dieser Reihenfolge.

Die für $\lambda=373$ angesehene Linie ist recht hell auf der Platte uber den ganzen photographierten Teil des Nebels (etwa 12 Bogenminuten von Nord nach Sud) hinweg. Die ubrigen sind sehr schwach. Es ist möglich, daß etwa um $\lambda=360$ noch eine Linie steht, aber recht fraglich.

Vielleicht gelingt mir mit frischer Spiegelversilberung einmal eine kräftigere Aufnahme dieses interessanten Spek. trums.

Astrophysikalisches Institut Königstuhl-Heidelberg, 1908 Aug. 17.

Max Wolf.

Elemente des VIII. Jupitermondes.

(Telegramm aus Cambridge, Mass, vom 20. August 1908.)

Crawford telegraphs the following elements of Jupiter VIII, computed by Crawford and Meyer:

$$
\begin{aligned}
& T=1908 \text { Aug. } 25.72 \text { Greenw. m. t. }
\end{aligned}
$$

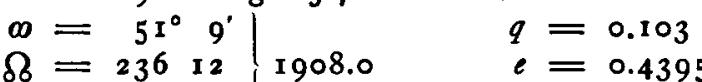

$$
\begin{aligned}
& i=14548 \quad \text { Period }=2_{2}^{2} .55
\end{aligned}
$$

Osculation r 908 March $8 \quad 19^{\mathrm{h}} 45^{\mathrm{m}} \cdot 5$ Greenw. m. t. 